\title{
Linx
}

Revue des linguistes de l'université Paris X Nanterre

$72 \mid 2015$

Former à l'écrit universitaire, un terrain pour la linguistique?

\section{Impact de discussions métalinguistiques sur l'apprentissage de la production écrite en allemand, langue étrangère}

\section{Eva Schaeffer-Lacroix}

\section{OpenEdition}

\section{Journals}

Édition électronique

URL : http://journals.openedition.org/linx/1676

DOI : $10.4000 /$ linx.1676

ISSN : 2118-9692

\section{Éditeur}

Presses universitaires de Paris Nanterre

Édition imprimée

Date de publication : 1 septembre 2015

Pagination : 233-237

ISSN : 0246-8743

\section{Référence électronique}

Eva Schaeffer-Lacroix, «Impact de discussions métalinguistiques sur l'apprentissage de la production écrite en allemand, langue étrangère », Linx [En ligne], 72 | 2015, mis en ligne le 01 mars 2016, consulté le 19 avril 2019. URL : http://journals.openedition.org/linx/1676 ; DOI : 10.4000/linx.1676 


\title{
Impact de discussions métalinguistiques sur l'apprentissage de la production écrite en allemand, langue étrangère
}

\author{
Eva SCHAEFFER-LACROIX \\ Groupe de recherche CoVariUs (Contextes, Variation, Usages) \\ Université de Paris-Sorbonne - IUFM de Paris
}

\section{Résumé}

Nous défendons l'idée que l'apprentissage de la production écrite en langue étrangère peut être soutenu à l'aide d'activités pédagogiques inspirées de méthodes du domaine de la linguistique, à savoir les discussions métalinguistiques et l'exploration de corpus numériques. Ces activités peuvent contribuer à faire émerger les représentations des apprenants, déclenchées par des marqueurs linguistiques (Culioli, 2002 : 172), et à les modifier si nécessaire (Matthey, 2010). Nous montrerons comment nous avons intégré ce type d'activités dans un scénario d'enseignement-apprentissage de l'allemand, basé sur l'exploitation d'un corpus contenant le script de film d'une série télévisée. La tâche principale du projet a été d'inventer une scène alternative ou supplémentaire pour le script de film. Nous présenterons l'analyse des données qui consiste à identifier des indices permettant de décrire l'impact des discussions métalinguistiques sur l'apprentissage de la production écrite, notamment l'exploration du domaine de la polyvalence catégorielle et l'élargissement du répertoire verbal.

Mots-clés

Production écrite, discussion métalinguistique, corpus, script de film, particules verbales, prépositions.

\section{Introduction}

\subsection{Problématique}

Quels repères linguistiques peut-on offrir aux apprentis-scripteurs en langue étrangère (LE) ? Nous partons de l'idée que la production écrite peut être soutenue à l'aide de méthodes inspirés de la linguistique, à savoir l'interaction verbale, pouvant se matérialiser dans des discussions métalinguistiques et l'exploration de corpus permettant d'aborder les textes d'experts de façon rationnelle et modulable. Nous espérons ainsi faire émerger les représentations des apprenants dans le domaine linguistique retenu pour le projet d'écriture. La verbalisation de ces représentations peut être déclenchée par l'observation de certains marqueurs linguistiques (Culioli, 2002 : 172). Ce procédé permettra de les modifier si elles se révèlent non pertinentes.

Les discussions métalinguistiques sont des échanges verbaux sur le fonctionnement de la langue (Trévise, 1994). Quel rôle peuvent-elles jouer lors d'un projet de production écrite en LE ? Influencent-elles principalement la qualité du produit (pertinence du texte en termes de sémantique, syntaxe, etc., évaluée à partir des caractéristiques linguistique de genres textuels)? Peut-on également observer une évolution dans le domaine du processus d'apprentissage (compétences pouvant être associées aux représentations mentales, par exemple, dans le domaine du raisonnement, de la catégorisation, etc.) ?

\subsection{Choix du genre textuel}


Nous avons choisi comme support de travail le script de film de la série télévisée Weissensee (Hess, 2010). Ce genre textuel contient deux parties principales distinctes, les dialogues et les didascalies. Les indications contenues dans les didascalies sont destinées à faire agir d'une certaine façon ceux qui tournent (dans) le film. Le visionnement du film pendant la formation fournit des indices, dont certains extralinguistiques, pouvant soutenir l'interprétation du sens des marques linguistiques repérées dans le script.

\subsection{Choix des points linguistiques}

Bronckart (1997 : 79) définit le genre textuel comme un «modèle social » ou un « exempletype ». L'inscription du projet dans ce domaine permet d'identifier les faits linguistiques correspondant à des besoins de formation. Dans le cas du script de film, il paraît pertinent de retenir le domaine de la sémantique des unités pluricatégorielles pouvant servir soit de particule verbale, soit de préposition, comme auf dans ces lignes de concordance du corpus bilingue Weissensee.

Gaucke legt die Fotos auf den Tisch, Gaucke pose les photos sur la table, il se lève, steht auf, er tritt ans Fenster, sieht hinaus. $\quad$ il se pose devant la fenêtre et regarde dehors.

En raison de leur fréquence élevée dans les didascalies, ces éléments peuvent être considérés comme constitutifs pour cette partie du script. De plus, Krause (2004) constate que les prépositions et, par extension, aussi les particules verbales ayant une même forme graphique que celles-ci, sont peu représentées dans les grammaires dites «pédagogiques », peut-être en raison d'une description et d'une analyse linguistique encore à affiner.

\section{Arrière-plan théorique}

\subsection{Linguistique de corpus}

On distingue généralement entre deux grands types de corpus numériques. Les corpus du premier type contiennent des échantillons de langue et visent à représenter une langue dans sa globalité. Les corpus du deuxième type contiennent des documents entiers représentant un genre textuel en particulier ; ils contiennent des écrits experts et renseignent ainsi sur les normes liées au genre textuel qu'ils représentent (Rastier, 2004). Nous avons opté pour l'usage de corpus contenant des textes entiers. À l'aide du système de gestion de corpus Sketch Engine (Kilgarriff, Rychly \& Pomikalek, 2011), nous avons transformé le script de Weissensee et notre traduction de son premier épisode en deux corpus étiquetés ${ }^{1}$. Le corpus monolingue et le corpus bilingue sont interrogeables en ligne à l'aide du concordancier de Sketch Engine.

\subsection{Sémantique des particules verbales et des prépositions}

Les particules verbales allemandes posent un certain nombre de problèmes pour l'interprétation. Elles peuvent être confondues avec des représentants d'autres catégories (prépositions, adverbes, etc.), et certaines d'entre elles, comme zu, possèdent un haut degré de polysémie : zuknöpfen [boutonner], zuhören [écouter], auf etwas zugehen [s'approcher de quelque chose], etc. Dans son article sur les unités polysémiques, Kravchenko-Biberson (2011) s'intéresse aux cadres théoriques permettant d'étudier la sémantique des préfixes russes et des particules verbales anglaises. Elle présente les apports interprétatifs de la théorie des

\footnotetext{
${ }^{1}$ On peut objecter que le corpus bilingue ne représente pas le script de film entier. En effet, il serait idéal de traduire la totalité du script par la suite.
} 
opérations énonciatives (TOE). Cela a réconforté notre choix d'opter pour une entrée par des concepts de type «invariant» (Culioli, 2002), proposés par Gilbert (2005), à savoir l'identification, la différenciation et la rupture. Ces concepts d'un haut degré d'abstraction sont en nombre suffisamment restreint pour un public d'apprenants de LE, et ils sont susceptibles d'accueillir une diversité d'interprétations des marques linguistiques.

\subsection{Discussions métalinguistiques}

Les discussions métalinguistiques, portant sur le fonctionnement de la langue, peuvent soutenir la formation de concepts scientifiques (Vygotski, 1934 : chapitre 6) et ainsi être mises à profit pour l'apprentissage d'une LE (Trévise, 1994). Pourquoi mener de telles discussions lors d'un projet de production écrite ? Peut-on défendre l'idée qu'échanger au sujet de faits de langue aiderait à comprendre ces faits et à les manipuler de façon pertinente pendant la production? Dans un cadre interactioniste, Matthey (2010) évoque «l'impact des relations humaines sur les formes langagières », et elle précise que l'interaction verbale peut être le lieu, le moyen et l'objet d'acquisition de la langue. Gombert (1996) présente les connaissances métalinguistiques comme un facteur qui entre en ligne de compte lors de l'apprentissage d'une LE. Il trouve opportun de soutenir les apprenants dans l'emploi qu'ils peuvent faire de telles connaissances, et il établit une interdépendance entre les tâches de production écrite et les connaissances métalinguistiques : les unes conditionneraient la qualité des autres.

\section{Choix didactiques et méthodologiques}

\subsection{Accès direct aux données linguistiques}

Le concordancier permet aux apprentis-scripteurs d'interroger directement un corpus dans le but de comparer leurs formulations à celles pour lesquelles des experts ont opté dans un énoncé ou une situation comparables. Il est également possible de consulter un corpus pour trouver une réponse à des interrogations sur le fonctionnement de la langue suscitées par la lecture d'un texte en LE (cf. 5.1.). Dans ce cas, l'apprenant est susceptible de procéder à la manière d'un « chercheur », voire d'un « linguiste » (Johns, 1991).

\subsection{Intégration dans un scénario}

Pourquoi avoir fait le choix d'intégrer les discussions métalinguistiques et le travail sur corpus dans un scénario d'enseignement-apprentissage ? Le sens se construit de façon complexe. Quand on cherche à comprendre un fait de langue, il ne suffit pas toujours de regarder une ligne de concordance isolée et figée : il convient de tenir également compte du cotexte qui doit parfois être élargi pour permettre une interprétation adéquate (Cislaru \& Sitri, 2009). Les contextes discursif et extra-linguistique peuvent également fournir des éléments d'interprétation des marqueurs linguistiques. Les indications d'action et d'agencement de personnes ou choses fournies dans les didascalies sont mises en scène dans la version filmée de Weissensee. Le visionnement du DVD donne accès à des informations susceptibles de soutenir la définition du sens d'une particule ou préposition (cf. $\underline{6.3 .}$.).

\section{Le projet Weissensee}

\subsection{Description}


Le projet Weissensee a été développé pour un groupe de trois étudiantes, inscrites en Master 1 documentation à l'IUFM de Paris (Institut universitaire de formation des maîtres). Nous avons profité de ces conditions de travail particulières pour observer finement les interactions verbales. Les 24 heures de formation ont été distribuées sur neuf séances de deux heures en présentiel et six séances d'une heure, proposées à distance. Les séances en présentiel ont été consacrées à la compréhension et à l'expression (écrit et oral), en s'appuyant sur le DVD et la version papier du premier épisode du script de la série Weissensee (Hess, 2010). Tout au long de la formation, la tâche principale, la production d'un texte pouvant remplacer ou prolonger une scène du script de film, a été présente en toile de fond. Les séances à distance ont été entièrement dédiées à des discussions métalinguistiques, menées à partir d'énigmes linguistiques (cf. 5.2.) ou de passages que nous avions relevés dans les premières versions de textes produits par les apprenantes. Pour communiquer à distance, nous nous sommes servies de la fonction «commentaire» de la plateforme Edmodo (Borg \& O'Hara, 2008), des fonctions audio et clavardage de Skype (Microsoft Corp, 2012) et du traitement de texte collaboratif en ligne TitanPad (Renner, 2010). Un entretien filmé avec une didacticienne extérieure a clos le projet.

\subsection{Productions écrites}

Trois types de production écrite individuelle ont ponctué le projet. Les étudiantes ont participé à un pré- et un post-test qui a consisté à retranscrire le plus fidèlement possible les événements spatiaux d'un bref extrait de film d'animation. Elles ont effectué chacune deux tâches intermédiaires, la description d'un extrait de deux minutes du film Weissensee. La tâche principale, l'invention d'une scène pour le script de film, a été effectuée tout au long du projet. Les étudiantes ont été invitées à rédiger un premier jet et à le retravailler jusqu'à ce qu'il corresponde de façon suffisante aux exigences linguistiques et discursives du genre textuel. Chacune d'elles a produit trois versions (nommées «V0», «V1» et «V2»), en retravaillant les passages que nous leur avions signalés comme problématiques. Nous avons donné des indications plus ou moins explicites, parfois allant jusqu'à fournir un «modèle tout fait » de requête (cf. 5.2.) destiné à faire trouver des solutions alternatives à l'aide du corpus. Une des séances à distance a été dédiée à la révision collective de tels passages. Pour ce faire, l'outil TitanPad nous a aidées à travailler simultanément sur les passages de notre choix (cf. 5.3.).

\subsection{Activités métalinguistiques collectives}

Les types d'activité métalinguistique de la formation partent d'observations de la langue plus ou moins guidées. Les étudiantes ont eu l'occasion d'explorer librement le corpus en y repérant des passages qu'elles trouvaient bizarres ou incompréhensibles. Certains de ces passages faisaient ensuite l'objet d'une discussion collective en ligne afin de trouver ensemble des explications. Un guidage modéré a été offert sous forme d'énigmes linguistiques, donnant l'occasion de réfléchir à partir de lignes de concordance à la polysémie de particules verbales sélectionnées.

\section{Analyse des discussions métalinguistiques}

\subsection{Discussions à partir d'observations libres du corpus}

Les observations libres ont permis de faire émerger des représentations sur la langue et de les réajuster si nécessaire. Dans l'extrait de discussion présenté ci-dessous, les étudiantes essaient 
de circonscrire le sens de la particule « entgegen » [vers, à la rencontre de].

Consigne : «Copiez dans TitanPad (...) des passages que vous trouvez peu clairs ou bizarres. »

$\begin{array}{ll}\text { Cornélia }^{2} & \begin{array}{l}\text { Martin geht Marion mit Lisa an der Hand entgegen.= je suis interessée } \\ \text { pour comprendre le « entgegen » }\end{array} \\ \text { Enseignante } & \text { Quelqu'un a une piste là ? Comprenez-vous « gegen »? } \\ \text { Marjorie } & \text { C'est « contre ». Et « ent », c'est un privatif, non? } \\ \text { Enseignante } & \begin{array}{l}\text { Oui, cela peut vouloir dire ça, mais ici, difficile de reconstruire un sens } \\ \text { privatif. Imaginez la scène : Martin et Lisa debout face à Marion... }\end{array} \\ \text { Solène } & \text { Ca pourrait vouloir dire aller à la rencontre de? } \\ \text { Enseignante } & \text { Super, Marjorie! Et bizarre, on a «contre » dedans aussi. } \\ \text { Marjorie } & \text { Non, en jaune }{ }^{4}, \text { c'est Solène! } \\ \text { Enseignante } & \text { Super Solène alors ! }\end{array}$

Marjorie définit d'abord ent- à l'aide d'un terme métalinguistique pertinent («privatif »), mais nécessitant d'autres explications afin de devenir une indication opérationnelle. Ensuite, nous faisons appel au contexte extralinguistique ce qui permet à Solène de formuler une traduction ${ }^{5}$ acceptable.

\section{2. Énigmes linguistiques basées sur des lignes de concordance}

Les catégories « rupture » et «différenciation » décrites par Gilbert (2005) sont à l'origine de deux énigmes linguistiques proposées lors du projet. La première énigme a servi à affiner la différence sémantique entre les particules verbales los, weg et ab (notion de rupture). À partir d'un choix de lignes de concordance imprimées, présentant des lacunes à la place des particules verbales, les étudiantes ont choisi, parmi les particules proposées dans la consigne, celle qui pouvait compléter le texte. Le résultat obtenu grâce à la discussion est particulièrement intéressant pour ab qui n'a pas les mêmes traits sémantiques en tant que particule verbale que comme préposition. En tant que particule verbale, il peut avoir la fonction de borne ouverte à gauche et à droite (début ou fin d'une action), mais comme préposition, il offre uniquement une ouverture vers la droite.

\begin{tabular}{|r|l|l|}
\hline arrêter de parler & ab/brechen & \\
\hline arrêter de rouler tout droit & ab/biegen & \\
\hline & ab/fahren & partir (en respectant un horaire) \\
\hline & ab/hauen & se casser, se tirer \\
\hline
\end{tabular}

La deuxième énigme s'est intéressée aux facettes d'unités pouvant être regroupées dans la catégorie «différenciation», comme auf et zu. La consigne suivante a invité le groupe à explorer les occurrences de la particule verbale zu dans le corpus monolingue Weissensee, en se servant de la requête préformatée suivante.

Dans corpus WeissenseeGermanAligned, observer :

\footnotetext{
${ }^{2}$ Les prénoms sont modifiés afin de garantir l'anonymat.

${ }^{3}$ L'orthographe des étudiantes a été maintenue.

${ }^{4}$ Code couleur sur TitanPad.

${ }^{5}$ En situation d'apprentissage de LE, on est parfois amené à appeller «traduction » ce qui correspondrait à une glose en langue maternelle (Culioli, $2002: 68$ ).
} 

$\mathrm{zu}$. sort left ${ }^{6}$
$\mathrm{zu}$, sort left
zu \& . (right)
Qu'est-ce que vous remarquez concernant zu?

La discussion qui a suivi l'exploration des lignes de concorance a enclenché une interrogation concernant la différence entre auf et zu.

Enseignante Que pensez-vous du sens de «zu»dans ces exemples : «Julia sieht ihm dabei zu. » [Julia le regarde faire.] ${ }^{7}$ et «Julia steht in der Tür und hört zu. »[Julia est dans l'encadrement de la porte et écoute.] ?

Solène zusehen et zuhören, le zu donne une précision dans le sens écouter et regarder qui se différencie d'entendre ou voir.

Marjorie Aucune idée, j'y ai réfléchi, mais comme pour le auf de aufpassen, je ne sais pas. Ah, finalement je suis d'accord avec Solène.

Enseignante Oui, une précision, mais dans quel sens ?

Marjorie Dans l'idée d'une direction, d'un but précis.

Enseignante Oui. Et contrairement à « auf » (« ich gehe auf ihn zu »), le but est... ? (je vous souffle le mot, je crois...). Non ? Il y a l'idée que l'on va atteindre ce but vraiment. Pour « auf », il y avait juste la direction. Et cela va aussi avec l'idée de fermeture, en quelque sorte (?? un peu tiré par les cheveux ?).

Cornélia oui on retrouve l'idée de determination

Enseignante Je ne comprends pas.

Cornélia =par rapport à la volonté de vraiment atteindre son but

Enseignante Ah oui, là, d'accord.

Nous avons retenu de cette discussion le concept d'implication, correspondant au terme de « détermination », et celui de cible. Avec auf, la cible est visée, mais non atteinte, tandis que zu marque le fait que la cible est atteinte.

Figure 1 - Corpus Weissensee, Sketch Engine, auf combiné avec zu vers sa droite.

\begin{tabular}{|r|c|l|}
\hline betreten zwei Kundinnen das Geschäft. Julia geht & auf & die beiden zu. JUIA Guten Tag. \\
\hline macht sich Julia von Martin los, sie geht & auf & Falk zu und packt ihn am Kragen. \\
\hline \multicolumn{3}{|c|}{} \\
\hline [deux clientes entrent dans le magasin. Julia se dirige & vers & elles Ø. JLIA Bonjour.] \\
\hline [Julia se libère de Martin, elle se dirige & vers & Falk Ø et l'attrape par le col.] \\
\hline
\end{tabular}

\subsection{Révision de productions écrites}

Afin de maintenir l'imbrication du discours lors de cette séance sur TitanPad, destinée à réviser deux passages de la version V2 de Solène sur Titanpad, nous insérons les initiales juste au début des tours de parole. Le discours n'est pas linéaire ; la révision *zuhören > an/hören proposée par Solène n'apparaît que vers la fin de la discussion.

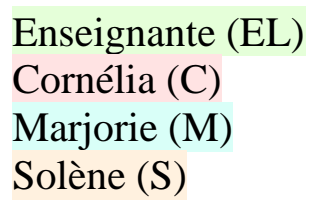

\footnotetext{
${ }^{6}$ Indications correspondant à l'interface du concordancier de Sketch Engine.

${ }^{7}$ Les traductions entre crochets « $[. .]$.$» ont été faites pour les besoins de l'article.$
} 
(EL) Que pourrions-nous dire à Solène concernant cela ?

Ich habe die Kassette zugehört... [J'ai écouté la cassette.] (S) anhören)

(C) Peut être revoir la particule verbale, en fonction de ce qu'on a dit en cours. Ou bien utiliser Pons pour les différences entre écouter/entendre (S) ce qui est bizarre c'est que je trouve bien le sens écouter pour zuhören?

La discussion porte ici sur le lien entre le choix de la particule et le sens du verbe.

(EL) Julia vorgibt zu sein beschäftigt. [Julia prétend être occupée.]

(C) L'ordre des mots n'est pas bon. (S) oui je viens de m'en rendre compte!

Julia (C) gibt beschäftig zu sein vor (je ne suis pas sûre)

(M) Julia gibt vor, beschäftigt zu sein.

Dans l'exemple ci-dessus, la révision nécessite de reconnaître que l'on a affaire à une particule séparable et de la placer à la fin d'un énoncé déclaratif qui n'est pas forcément marqué par un point final. Solène et Cornélia verbalisent chacune une forme de prise de conscience, sans arriver à un résultat de révision totalement pertinent. Il sera finalement apporté par Marjorie.

\section{Impact sur l'apprentissage de la production écrite}

\subsection{L'exploration du domaine de la polyvalence catégorielle}

Dans le cadre de son étude sur les particules verbales, Kravchenko-Biberson (2011) évoque la problématique de la polyvalence catégorielle. Elle précise que les unités concernées ont un sens prototypique stable, tout en offrant une polysémie importante en situation d'instanciation. Elle relève aussi la productivité des particules verbales : les locuteurs peuvent créer de nouveaux verbes à particule et interpréter ceux créés par d'autres (cf. l'invention lexicale mentionnée en $\underline{6.2 .}$ )

\subsubsection{Particule verbale ou préposition ? (Solène)}

Des phénomènes de non-pertinence catégorielle et/ou sémantique existent dans toutes les versions de la scène inventée par Solène.

JULIA (sie wirft *eine Blick *draußen zu.) > nach draußen

[Elle jette un regard vers l'extérieur.] (V2)

Die Tür von dem Musikladen *sich öffnet auf. > öffnet sich ; > geht auf

[La porte du magasin de musique s'ouvre.] (V2)

Dans le premier exemple ci-dessus, la préposition nach [vers] manque, et la particule zu est superflue. Elle serait pertinente si le verbe se référait à un être vivant et non à un lieu : sie wirft ihm einen Blick zu [elle lui jette un regard]. Dans le deuxième exemple, la particule auf complète un verbe pronominal qui se construit en principe sans particule. Il serait toutefois possible de conserver auf en choisissant le verbe auf/gehen [s'ouvrir].

Dans l'extrait suivant d'un texte de Cornélia, proposé sur TitanPad pour en discuter, la particule an est également superflue. 
Enseignante Que pourrions-nous dire à Cornélia (V2) concernant cela ? «Hans war immer schlecht angekleidet. »

Solène (peut être: faire une requête dans sketch Engine: trouver d'autre prépositions)

Solène conseille ici à Cornélia de remplacer la particule an par ce qu'elle appelle « une autre préposition ». Elle semble d'ailleurs avoir conscience de ses difficultés dans le domaine de la polyvalence catégorielle, comme l'illustre l'échange suivant.

Solène

Martin steht auf. Tout à l'heure, auf marquait le lieu. Ici... Ah non, c'est une particule. J'ai du mal à différencier, des fois.

Enseignante Oui, certaines particules et les prépositions mènent une double vie... Mais auf est quand même spatial.

\subsubsection{Explorer des propriétés catégorielles de an (Marjorie)}

L'élément an [₹à], faisant partie des éléments que l'on peut regrouper sous l'étiquette « différenciation », est absent dans les trois premiers textes de Marjorie, donc le pré-test et les deux tâches intermédiaires et, après rature, aussi dans le post-test. Les deux textes de type «tâche intermédiaire » contiennent le verbe $* \varnothing /$ gucken [regarder] qui devrait être doté de la particule an : an/gucken. Les versions de la scène inventée attestent d'abord d'un emploi superflu de la particule an (dans an/probieren > probieren [essayer]), ensuite d'emplois pertinents du point de vue sémantique et syntaxique à côté d'emplois non pertinents. Des erreurs sémantiques (an > auf) côtoient des erreurs de catégorie : Marjorie remplace deux fois une terminaison de datif (cas marquant le bénéficiaire d'une action) avec an suivi d'un groupe nominal : «Hans zeigt eine Foto an der Mann » [Hans montre à l'homme une photo] au lieu de «Hans zeigt dem Mann ein Foto ». Elle utilise ainsi an comme une préposition. Dans le post-test, nous trouvons la formulation suivante, pouvant correspondre à «Quand elle le regarde de nouveau, ... ».

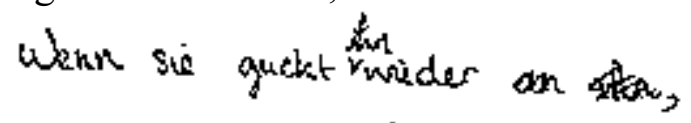

L'élément an apparaît ici d'abord sous forme de préposition : «Wenn sie guckt wieder an ihn » [Quand elle regarde de nouveau « vers lui »]. Il est ensuite barré, et le pronom ihn est déplacé : «Wenn sie guckt ihn wieder,... », au lieu de «Wenn sie ihn wieder anguckt, ... ». Après une phase d'exploration et d'hésitation sémantique et catégorielle, an disparaît de nouveau, mais pas dans tous les contextes : nous trouvons dans le post-test l'énoncé suivant qui est pertinent du point de vue du verbe à particule (il s'agit, certes, d'un verbe très répandu) : «Die Mädchen ruft Jack-Jacks Eltern an » [La fille appelle les parents de JackJack].

Figure 2 - Emplois de an dans tous les textes de Marjorie. 


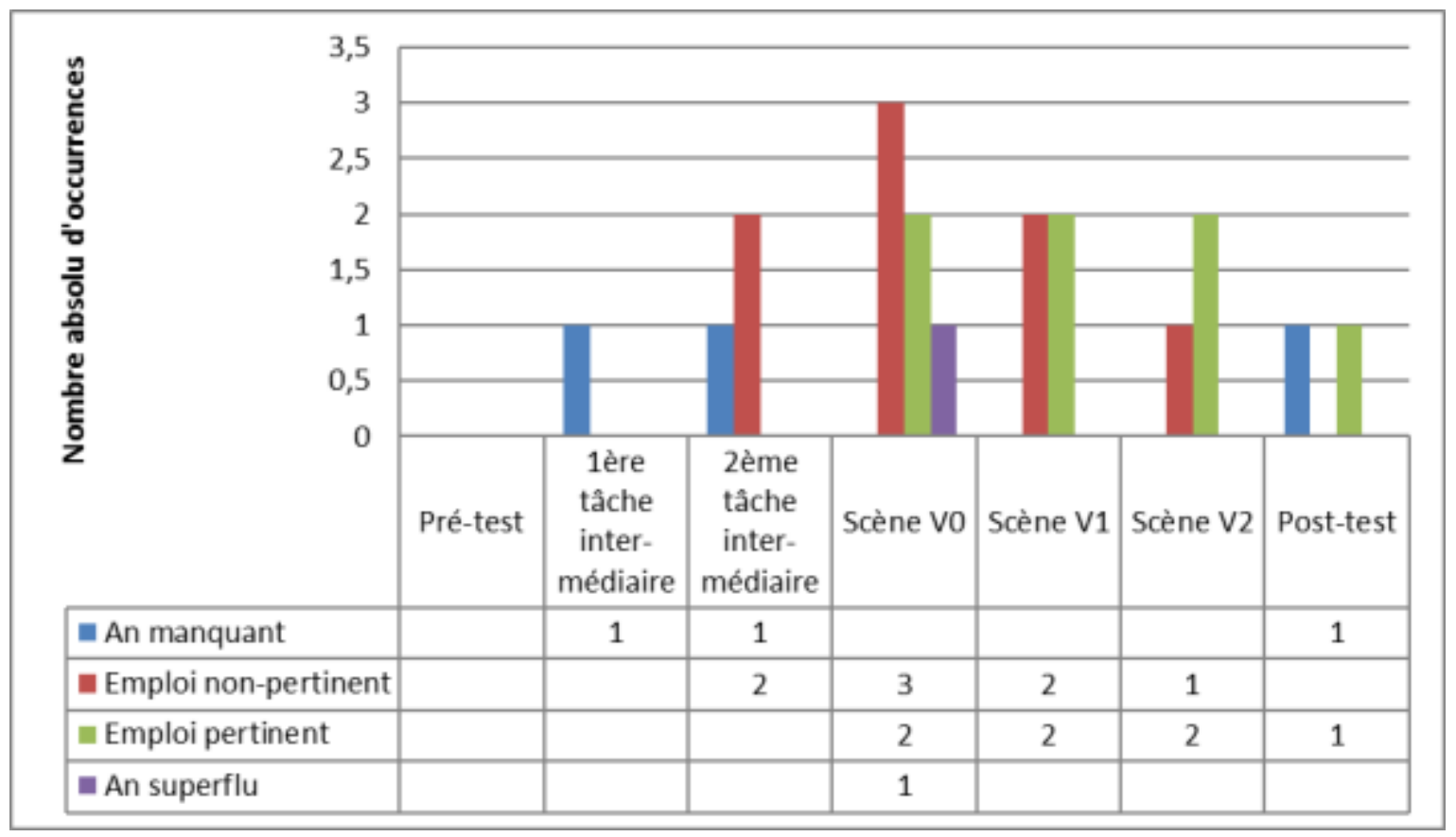

Selon ce graphique, une «maîtrise » croissante de an peut être observée dans les textes de Marjorie, même si son tâtonnement catégoriel et sémantique est encore sensible vers la fin du projet.

\subsection{L'élargissement du répertoire verbal}

En utilisant le terme de « répertoire verbal », nous souhaitons rendre compte d'une évolution que nous avons observée dans les textes des apprenantes, à savoir l'augmentation du nombre d'unités pluricatégorielles et leur emploi dans une plus grande variété de situations. Boutet et Deprez (2002 : 37) donnent à ce terme une acception qui nous semble en accord avec la pratique de discussions métalinguistiques.

On peut (...) envisager les répertoires [verbaux] d'un point de vue dynamique (...) comme le résultat de processus de construction, mis en œuvre et négociés par les locuteurs au sein des interactions sociales.(...). Ce travail dynamique (...) porte sur les formes linguistiques elles-mêmes : inventions lexicales, emprunts, (...).

On peut rapprocher l'idée d'emprunt de la pratique d'écriture intertextuelle de Cornélia et de Solène (cf. $\underline{6.3}$.).

\subsubsection{Variété de situations (Cornélia)}

Cornélia semble avoir trouvé le travail métalinguistique cognitivement exigeant. Toutefois, dans l'entretien, elle utilise des termes qui suggèrent que dans une certaine mesure, elle a compris et appliqué des concepts clés de la TOE relevant de l'interprétation sémantique, comme la variation, l'interieur et l'extérieur.

Cornélia

Enquêtrice on est restées pas mal autour des mêmes [particules] parce qu'il y avait tellement de + variations. Du coup, et puis comme en tout cas, je n'ai pas un niveau + (rit)...

Oui? 
Il est vrai que les textes de Cornélia contiennent une proportion élevée de certains éléments en particulier, notamment de an. La scène 43 du script de Weissensee dont s'inspire Cornélia pour rédiger, débute avec un passage contenant quatre fois an, dans des situations variées (temps, lieu, «métaphore »), comme préposition ou particule verbale.

Später am Abend. Dunja sitzt am Flügel und arbeitet an einem Lied...

DUNJA (singt leise) (...)

Dunja feilt an den Harmonien, probiert aus... (...)

JULIA Hast du nicht auch manchmal das Gefühl, als ob du erstickst?

DUNJA Nein, vorher fange ich an zu singen.
Plus tard le soir. Dunja est assis au piano à queue et retravaille une chanson...

DUNJA (chante doucement) (...)

Dunja peaufine les harmonies, fait des essais... (...)

JULIA N'as-tu pas parfois l'impression d'étouffer ?

DUNJA Non, avant, je me mets à chanter.

Le graphique suivant dessine l'évolution d'un emploi restreint de an vers une utilisation assez fréquente dans la scène inventée, caractérisée par une large proportion d'emplois pertinents. Nous n'avons écarté ici les occurrences coprésentes dans le script original et les scènes de Cornélia.

Figure 3 - Emplois de an dans tous les textes de Cornélia.

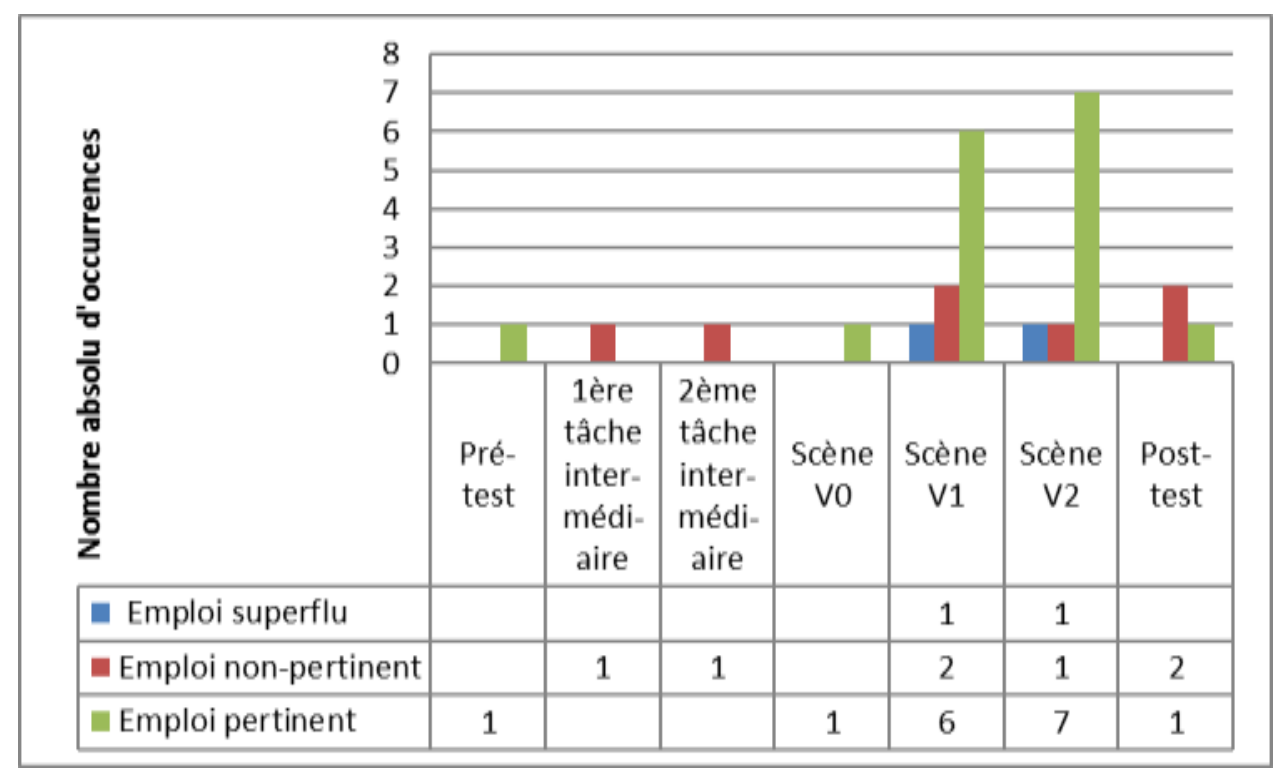

Trois situations peuvent être identifiées pour an : un emploi «métaphorique » (huit variantes de l'expression an jn/etw denken [penser à quelqu'un/quelque chose]), un emploi temporel (Am nächsten Tag [le jour suivant]) et un emploi spatial (am Weissensee entlang [le long du lac Weissensee]). Le choix du procédé d'écriture largement intertextuelle (cf. 6.3.) semble avoir aidé Cornélia à introduire an, un élément particulièrement abstrait, polysémique et pluricatégoriel, dans son répertoire verbal et à s'approprier certaines de ses propriétés.

\subsubsection{Variété d'éléments (Solène)}

Comme l'illustre le tableau suivant, entre le pré- et le post-test, Solène a élargi son répertoire 
de particules et/ou de prépositions aussi bien du point de vue quantitatif que qualitatif. Vers la fin du projet, la maîtrise des propriétés liées à l'appartenance des éléments à la catégorie « particule » ou «préposition » lui pose encore problème (pour an et durch), mais elle arrive à appliquer la particule zu non seulement dans une situation qui a été vue lors d'une discussion, à savoir zu/hören [écouter attentivement], mais aussi dans une situation comparable, à savoir $\mathrm{zu} / \mathrm{sehen}$ [regarder attentivement] (cf. 6.1.). Notons que ab et zu ont été observés dans le contexte d'énigmes linguistiques du projet et que le sens de an a été abordé lors d'une séance de clavardage sur Edmodo.

Tableau 1 - Évolution de Solène entre le pré- et le post-test.

\begin{tabular}{|l|l|l|l|l|}
\hline & \multicolumn{2}{|l|}{ Pré-test } & Post-test \\
\cline { 2 - 5 } & choix pertinent & choix non pertinent & choix pertinent & choix non pertinent \\
\hline ab & & & particule & \\
\hline an & préposition & & préposition & $\begin{array}{l}\text { préposition *an > mit } \\
\text { (sémantique) }\end{array}$ \\
\hline durch & & particule (syntaxe) & & $\begin{array}{l}\text { emploi d'une particule } \\
\text { non séparable comme } \\
\text { particule séparable, } \\
\text { ensuite comme } \\
\text { préposition (catégorie) }\end{array}$ \\
\hline um & & particule (syntaxe) & préposition & \\
\hline pu & & particule manque & $\begin{array}{l}\text { particule } \\
\text { particule }\end{array}$ & \\
\hline
\end{tabular}

6.3. L'écriture intertextuelle, une méthodologie à retenir ?

Dans l'entretien final, Marjorie et Solène évoquent le contexte, donc la connaissance du script de film, mais aussi du film visionné en cours, comme aide à la production écrite.

Marjorie : Moi, j'ai trouvé ça intéressant, parce qu'on avait déjà + c'était plus simple, en fait, parce qu'on avait déjà un contexte, on avait déjà des personnages, + on ne partait pas de de rien, et du coup, ça + permet de de donner plus de de corps au texte car c'est moins + moins banal.

Enquêtrice : Mhm.

Solène : Oui, finalement, on s'éloignait au fur et à mesure. Au début, on reste proche du texte, et finalement, on change tout, oui, et ça change + le texte complètement.

Dans sa version V0, Solène reste, en effet, très «proche du texte » source. Elle s'insère intimement dans la scène 24 pour finalement en écrire une suite très personnelle. Ce procédé intertextuel mène à une proportion élevée de formes pertinentes dès la version $\mathrm{V} 0$ ce qui, nous l'admettons, ne garantit pas en soi l'acquisition des formes. L'observation de l'emploi que Solène fait des unités pluricatégorielles permet de se prononcer sur le potentiel d'acquisition de cette méthode.

Figure 4 - Formes pluricatégorielles variées dans les textes de Solène. 


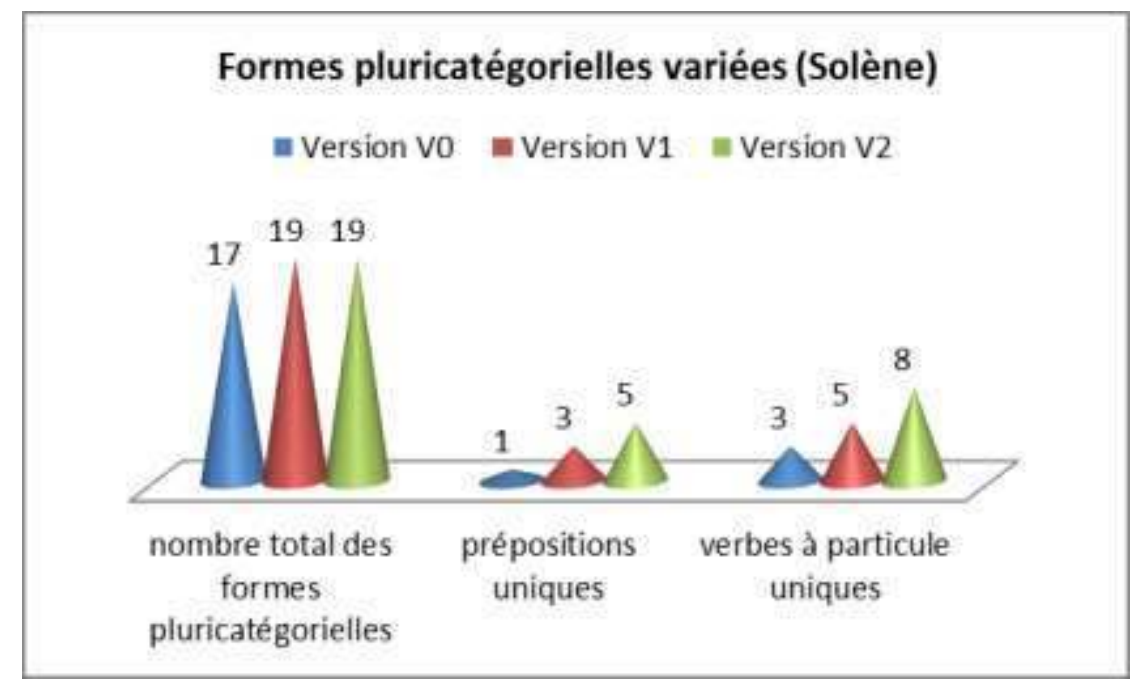

La première colonne du graphique ci-dessus représente l'évolution du nombre total des prépositions et particules verbales pluricatégorielles variées dans les trois versions du texte de Solène. Les deux autres colonnes se concentrent sur les éléments uniques, donc ceux qui n'apparaissent pas dans la scène 24 du script original. Les éléments non-pertinents sont également comptabilisés.

\begin{tabular}{|l|l|l|l|}
\hline Non-pertinence & V0 & V1 & V2 \\
\hline syntaxique & 1 & & 1 \\
\hline sémantique & & 1 & 1 \\
\hline catégorielle & & & 1 \\
\hline
\end{tabular}

Avec l'adoption d'une écriture plus libre, moins adossée au script existant, les erreurs deviennent plus fréquentes. Elles témoignent d'un suremploi des propriétés des particules au détriment de celles des prépositions (cf. 6.1.1.). Tout n'est pas encore stabilisé au niveau de l'apprentissage dans le domaine observé. Toutefois, au fil des trois versions, Solène a réussi à clairement enrichir son répertoire de particules verbales $(3>8)$ et de prépositions $(1>5)$ variées et uniques, donc présentes uniquement dans son texte. La technique intertextuelle semble avoir pour elle, tout comme pour Cornélia, un « potentiel acquisitionnel » certain.

\section{Conclusion}

Les discussions menées en présentiel ou à distance, ainsi que les textes produits par les trois apprenantes permettent de constater deux apports principaux de la méthodologie proposée. Premièrement, les données contiennent des indices d'évolution dans le domaine de la polyvalence catégorielle : les étudiantes explorent les propriétés syntaxiques et/ou sémantiques d'un terme polysémique, elles progressent, toutefois sans forcément arriver à un résultat d'acquisition définitif. Deuxièmement, les données attestent d'un élargissement du répertoire des unités observées, autant du point de vue quantitatif que du point de vue qualitatif (variations sémantiques et situationnelles). Les progrès ne se restreignent pas aux éléments ayant fait l'objet d'une discussion explicite ou intense : an n'a été mentionné qu'en passant lors d'un échange sur Edmodo, et toutefois, c'est autour de cet élément que l'on constate des tâtonnements fructueux.

La prise en compte aussi bien du cotexte que du contexte semble avoir joué un certain rôle pour affiner le sens des particules et prépositions. La méthode d'écriture intertextuelle, choisie 
par deux des trois apprentis-scripteurs, a mené à des résultats convaincants au niveau de la pertinence linguistique et discursive des productions écrites. Weissensee a laissé une certaine marge de manœuvre aux étudiantes ce qui a permis à chacune de tirer profit de la formation à sa manière.

Weissensee est un projet qui semble avoir «bien fonctionné ». Toutefois, il convient de nuancer les résultats obtenus en rappelant que les participantes se distinguent d'autres types de public par des compétences professionnelles particulièrement adaptées au travail sur corpus. En tant que futures «professeurs documentalistes », elles savent explorer et annoter des textes stockés en ligne, et selon ce qu'elles disent dans l'entretien, elles voient l'intérêt de manipuler des outils numériques correspondant à ces activités. Par la suite, nous comptons proposer ce même type de projet à un groupe plus conséquent qui n'est pas spécialisé en documentation, informatique ou linguistique.

\section{Bibliographie}

BOUTET, J., DEPREZ, C., 2002, «Ici et là-bas, public et privé. Des catégories à interroger », Cahiers de l'Institut de Linguistique de Louvain, 28-3/4, 37-46.

BRONCKART, J.-P., 1997, Activité langagière, textes et discours. Pour un interactionisme socio-discursif, Paris, Delachaux et Niestlé.

CISLARU, G., SITRI, F., 2009, « Texte et discours. Corpus, co-texte et analyse automatique du point de vue de l'analyse de discours », Corpus 8, p. 85-104.

CULIOLI, A., 2002, Variations sur la linguistique. Entretiens avec Frédéric Fau. Bonchamplès-Laval, Klincksieck, 2009 pour la présente édition.

GILBERT, E., 2005, «Ébauche d'une formalisation des prépositions In, On et At », Cycnos 21(1) <http://revel.unice.fr/cycnos/index.html?id=11>

GOMBERT, J.-É., 1996, «Activités métalinguistiques et acquisition d'une langue », Acquisition et interaction en langue étrangère 8, p. 41-55. http://aile.revues.org/1224

HESS, A., 2010, Weissensee. Script de film. http://www.stichwortdrehbuch.de/drehbuch

JOHNS, T., 1991, "Should you be persuaded - two samples of data-driven learning materials », dans T. Johns, P. King (éds), (1991) Classroom concordancing. English language research journal 4. Birmingham, Birmingham University, p. 1-13.

KRAUSE, M., 2004, «Konkurrenz, Komplementarität und Kooperation im Bereich der Präpositionen und Verbalpartikeln oder Wie lange noch müssen Präpositionen und Verbalpartikeln in Grammatiken ein Schattendasein führen? », Linguistik online 18-I/04, p. 35-69.

KRAVCHENKO-BIBERSON, O., 2011, «Le modèle cognitif et la TOE : deux points de vue sur l'identité sémantique des unités polysémiques », CORELA <http://corela.edel.univpoitiers.fr/index.php?id=2024>

MATTHEY, M., 2010, «Interaction : lieu, moyen ou objet d'acquisition ? », dans C. Vargas, L.-J. Calvet, M. Gasquet-Cyrus, D. Véronique, R. Vion (éds), Langues et sociétés : Approches sociolinguistiques et didactiques. Paris, L'Harmattan, p. 31-42.

RASTIER, F., 2004, «Enjeux épistémologiques de la linguistique de corpus », Texto! http://www.revue-texto.net/Inedits/Rastier/Rastier_Enjeux.html

TREVISE, A., 1994, «Représentations métalinguistiques des apprenants, des enseignants et des linguistes: un défi pour la didactique », Bulletin VALS-ASLA (Association suisse de linguistique appliquée, 59, p. 171-190.

VYGOTSKI, L.S., 1934/1997, Pensée et langage. Paris, La Dispute. 


\section{Outils en ligne}

Borg, N., O'Hara, J., 2008, Edmodo. Plateforme d'apprentissage. http://www.edmodo.com Kilgarriff, A., Rychly, P., Pomikalek J. (nd) Sketch Engine.

http://www.sketchengine.co.uk/

Microsoft Corp., 2012, Skype. http://www.skype.com/intl/en/home

Renner, M., 2010, TitanPad. Traitement de texte collaboratif. http://titanpad.com/ 